\title{
ASYMPTOTIC REPRESENTATION FOR THE BLUMENTHAL-NEVAI ORTHOGONAL POLYNOMIALS IN THE ESSENTIAL SPECTRUM
}

\author{
Renato Spigler and Marco Vianello
}

\begin{abstract}
A recent Liouville-Green (or WKB)-type approximation theorem for linear second-order difference equations is used to study the asymptotic behavior of certain families of orthogonal polynomials in the essential spectrum. These polynomials fall in the class first investigated by $\mathrm{O}$. Blumenthal in 1898, and then studied extensively by P. Nevai in the late 70s. When explicit qualitative representations already are available, the WKB method also allows obtaining precise bounds for the error terms. This procedure is applied in detail to the case of Jacobi polynomials, for which error estimates are derived also in the parameter range $-1<\alpha, \beta \leq-1 / 2$. A second-order discrete WKB theory with error bounds then is developed and applied, for illustration, to the ultraspherical polynomials, thereby obtaining a representation with an estimated error of order $O\left(n^{-2}\right)$, also valid for $\lambda<0$.
\end{abstract}

\section{Introduction}

The widely celebrated Liouville-Green (or WKB) approximation for solutions of linear second-order differential equations has been known and used for many decades by physicists and put on sound (rigorous) mathematical bases by F. W. J. Olver in 1961, cf. $[19,20]$. The first extensions to difference equations seem to have appeared much later, in $[8,4,29]$. More recently, a discrete Liouville-Green (LG) theory for some classes of linear second-order oscillatory difference equations has been developed [22] where, in the spirit of Olver [20], precise error bounds accompany the asymptotic representations. In [10], a discrete WKB theory also has been developed following different lines. A unified LG theory has been presented in [23], to include both linear second-order differential and difference equations; in particular, certain nonoscillatory difference equations were treated there. All these results are part of a recently observed renaissance of the entire subject of difference equations; for a review of all results concerning the discrete WKB approximation, the reader is referred to [24]. For the case of linear second-order equations, in particular describing families of orthogonal polynomials, other asymptotic results (not of the LG-type) appeared in [14, 31].

The purpose of the present paper is threefold. First $(\S 2, \S 3.1)$, we use our LG theory to describe the asymptotic behavior of solutions of certain linear second-order difference equations, which include three-term recurrence equations satisfied by wellknown families of orthogonal polynomials, which form a subclass of the BlumenthalNevai polynomials, $[3,16]$. The latter includes the entire set of polynomials studied in [14]. The ensuing asymptotic representations hold in the essential spectrum.

Received July 7, 1995, revised April 25, 1996.

1991 Mathematics Subject Classification: 33A65, 39A10, 39A12, 65D20, 65Q05.

Key words and phrases: Liouville-Green (WKB) approximation, linear difference equations, orthogonal polynomials, Blumenthal-Nevai polynomials, Jacobi polynomials, ultraspherical polynomials. 
The second purpose (§3.2) is to obtain precise error estimates for the Darbouxtype asymptotic representations of Jacobi polynomials [25, Thm. 8.21.8]. Though for general Jacobi polynomials our estimates are uniform for $x=\cos \theta, \theta \in[\varepsilon, \pi / 2-\varepsilon] \cup$ $[\pi / 2+\varepsilon, \pi-\varepsilon]$, they cannot be applied at $x=0$. For the ultraspherical polynomials, however, they hold uniformly on $[\varepsilon, \pi-\varepsilon]$. Asymptotic formulae with bounds, valid in a half-interval up to the endpoint -1 or 1 (included), under some restrictions on $\alpha, \beta$, have been derived in the 1980s by Hahn [11], Frenzen and Wong [9], and Baratella and Gatteschi [2]. While such bounds are better than ours, our estimates hold without any restriction on the parameters, in particular for $-1<\alpha, \beta \leq-1 / 2$ (e.g., $\lambda<0$ for the ultraspherical polynomials), which is a case apparently not covered in the literature. Moreover, other than in our estimates, those of $[2,9]$ hold (under certain restrictions on the parameters) only on one half-interval. Under more severe restrictions, they hold on the whole interval, $[-1,1]$. We stress the fact that our estimates hold on the whole set $(-1,1) \backslash\{0\}$ (and uniformly on compact subsets of it) irrespective of the parameters for general Jacobi polynomials, and on $(-1,1)$ in the case of ultraspherical polynomials. Indeed, even the restriction $\alpha, \beta>-1$ (necessary for orthogonality) can be removed if one focuses on the polynomials merely as solutions to the "Jacobi difference equation."

In §3.3, finally, we develop a second-order discrete WKB approximation with error bounds, which is applied, for the purpose of illustration, to the ultraspherical polynomials, thus obtaining a representation with an estimated error of order $O\left(n^{-2}\right)$, valid also for $\lambda<0$.

\section{Asymptotic representation for the Blumenthal-Nevai orthogonal polynomials}

We first recall, as a basic device, that it is possible to take a rather general three-term recurrence equation such as

$$
Y_{n+2}+A_{n} Y_{n+1}+B_{n} Y_{n}=0, \quad n \in \mathbf{Z}_{\nu},
$$

where $\mathbf{Z}_{\nu} \equiv\{n \in \mathbf{Z}: n \geq \nu\}, \nu \in \mathbf{Z}$, and $A_{n} \neq 0$ in $\mathbf{Z}_{\nu}$, into the "canonical form"

$$
\Delta^{2} y_{n}+q_{n} y_{n}=0, \quad n \in \mathbf{Z}_{\nu}
$$

by the transformation

$$
Y_{n}=\alpha_{n} y_{n} ; \alpha_{n}=\alpha_{\nu+1} \prod_{k=\nu}^{n-2}\left(-\frac{A_{k}}{2}\right), \quad n \geq \nu+2,
$$

$\alpha_{\nu}$ and $\alpha_{\nu+1} \neq 0$ being arbitrary constants. Correspondingly, the coefficient $q_{n}$ in (2) is given by

$$
q_{n}=-1+\frac{4 B_{n}}{A_{n} A_{n-1}}, \quad n \geq \nu+1
$$

$q_{\nu}=\left[\Delta^{2} \alpha_{\nu}+\left(A_{\nu}+2\right) \Delta \alpha_{\nu}+\left(A_{\nu}+B_{\nu}+1\right) \alpha_{\nu}\right] / \alpha_{\nu+2}$, cf. [22].

In [22], we proved the following

Theorem 2.1 (Discrete Liouville-Green approximation). Suppose that equation (2) is given, with

$$
q_{n}=a+g_{n}, \quad a>0
$$


where

$$
\sum_{n=\nu}^{\infty}\left|g_{n}\right|<\infty .
$$

Then there exists $n_{0} \in \mathbf{Z}_{\nu}$ such that there are two linearly independent solutions to (2), having the form

$$
y_{n}^{ \pm}=\left(\lambda^{ \pm}\right)^{n}\left[1+\varepsilon_{n}^{ \pm}\right] \quad \text { for } n \geq n_{0}
$$

where

$$
\lambda^{+}=1+i a^{1 / 2}, \quad \lambda^{-}=\overline{\lambda^{+}}
$$

are the roots of the characteristic polynomial associated with (2) with $g_{n} \equiv 0$, and

$$
\begin{gathered}
\left|\varepsilon_{n}^{ \pm}\right| \leq \frac{V_{n}}{1-V_{n}}, \quad n \geq n_{0}, \\
V_{n}:=[a(1+a)]^{-1 / 2} \sum_{k=n}^{\infty}\left|g_{k}\right| .
\end{gathered}
$$

When $g_{n}$ is real valued, $y_{n}^{+}$and $y_{n}^{-}$are complex conjugate.

In proving Theorem 2.1 in [22], we have shown that $n_{0}$ can be chosen to be

$$
n_{0}=\min \left\{n: n \in \mathbf{Z}_{\nu}, V_{n}<1\right\} \text {. }
$$

Remark 2.2. One of the most important results in Theorem 2.1 is that of providing precise error bounds in the asymptotic representation of a basis for equation (2). This is in the spirit of Olver's approach to the Liouville-Green approximation for differential equations (cf. [20]). Moreover, even the purely qualitative behavior $y_{n}^{ \pm} \sim\left(\lambda^{ \pm}\right)^{n}$ in (7) could not be obtained by the classical Poincaré's or Perron's theorems, since $\left|\lambda^{+}\right|=\left|\lambda^{-}\right|$in (8), cf. [15].

In what follows, we shall assume $A_{n}$ and $B_{n}$ to be real, so that, in particular, $g_{n}$ and the transformation coefficient $\alpha_{n}$ in (3) will be real. Theorem 2.1 then implies, for every real solution to (1), the representation

$$
Y_{n}=\mathcal{A} \alpha_{n} \rho^{n}\left[\cos (n \theta+\eta)+E_{n}\right], \quad n \geq n_{0},
$$

where $\mathcal{A}$ and $\eta$ are two real parameters, and

$$
\begin{gathered}
\rho:=\left|\lambda^{+}\right|=(1+a)^{1 / 2}, \quad \theta:=\arg \lambda^{+}, \\
\left|E_{n}\right| \leq\left|\varepsilon_{n}^{+}\right|=O\left(V_{n}\right),
\end{gathered}
$$

provided that

$$
\lim _{n \rightarrow \infty} \frac{B_{n}}{A_{n} A_{n-1}}=: L>\frac{1}{4}
$$

$L=(a+1) / 4$, and

$$
\sum_{n=\nu+1}^{\infty}\left|\frac{B_{n}}{A_{n} A_{n-1}}-L\right|<\infty,
$$

cf. [22]. In fact, in terms of the coefficients of the original three-term recurrence equation, (15) and (16) correspond to the requirement that $q_{n} \rightarrow a>0$ and to the convergence of the series in (6), respectively. 
Remark 2.3. Note that all real solutions $y_{n}$ to (2), under the hypotheses of Theorem 2.1, are oscillatory according to the usual definition (e.g. [21, 22]). This can be shown from (12) (see Appendix 1), but it does not seem to be derivable immediately from the best known oscillation criteria $[1,13,21,22]$. The oscillatory behavior of $Y_{n}$, however, is certainly guaranteed when $A_{n}$ has constant sign for $n$ sufficiently large. In fact, if $A_{n}<0$ for all $n$ large enough, $\alpha_{n}$ in (12) has constant sign, while if $A_{n}>0$ for all $n$ large enough, $\alpha_{n}$ changes sign at each $n$, and the oscillations persist (cf. Appendix 1). Another important consequence of (12) is the growth estimate

$$
Y_{n}=O\left(\alpha_{n} \rho^{n}\right), \quad n \geq n_{0} .
$$

Hereafter, we shall focus our attention on families of monic orthogonal polynomials which are defined by three-term recurrence equations like

$$
P_{n+2}(x)-\left(x-\gamma_{n}\right) P_{n+1}(x)+\delta_{n} P_{n}(x)=0, \quad n=-1,0,1, \ldots,
$$

$P_{-1}(x) \equiv 0, P_{0}(x) \equiv 1, \gamma_{n}$ being real and $\delta_{n}>0$. We shall refer to monic orthogonal polynomials for which

$$
\lim _{n \rightarrow \infty} \gamma_{n}=\gamma, \quad \lim _{n \rightarrow \infty} \delta_{n}=\delta
$$

are both finite as the Blumenthal-Nevai class (see [7, p. 101]). Note that this case includes the Jacobi and the Pollaczek polynomials, for instance. Under these hypotheses, it is known that the Hamburger moment problem is determined and that, if the smallest and the largest limit-points of the spectrum are denoted by $\sigma$ and $\tau$, respectively, then

$$
\sigma=\gamma-2 \sqrt{\delta}, \quad \tau=\gamma+2 \sqrt{\delta} .
$$

Moreover, Blumenthal [3] showed that the zeros of the $P_{n}(x)$ are dense in $[\sigma, \tau]$, and Nevai proved that this interval is a subset of the spectrum (cf. [6, p. 172] and [7, pp. 122, 104]). Other deep investigations about spectral and asymptotic properties of the polynomials in the Blumenthal-Nevai class have been carried out by many authors (see, e.g., [5, 7, 16, 17, 18, 26, 27] and the references therein). Below, we shall restrict our attention to the asymptotic behavior as $n \rightarrow \infty$ for $x$ in the essential spectrum $[\sigma, \tau]$.

Comparing (18) with (1), one can see that in (15)

$$
L=L(x)=\frac{\delta}{(x-\gamma)^{2}}>\frac{1}{4}
$$

for all $x \in(\sigma, \tau) \backslash\{\gamma\}$. Note that $\delta \geq 0$ in (19), in any case. If $\delta=0$, condition (21) cannot be fulfilled and the interval $[\sigma, \tau]$ degenerates into the singleton $\{\gamma\}$. Moreover, since

$$
\alpha_{n}=\alpha_{n}(x)=\prod_{k=\nu}^{n-2}\left(\frac{x-\gamma_{k}}{2}\right)
$$

provided that $x \neq \gamma$, one can choose $\nu=-1$ as long as $x \neq \gamma_{k}$ for each $k$; otherwise, one takes $\nu=m+1$ where $m=\max \left\{k: \gamma_{k}=x\right\}$. We stress that, in any case, $\nu=\nu(x)$.

If condition (16) also is satisfied, which for the Blumenthal-Nevai class becomes

$$
\sum_{n=\nu+1}^{\infty}\left|\frac{\delta_{n}}{\left(x-\gamma_{n}\right)\left(x-\gamma_{n-1}\right)}-\frac{\delta}{(x-\gamma)^{2}}\right|<\infty
$$


then the asymptotic representation

$$
\begin{gathered}
P_{n}(x)=\mathcal{A}(x)\left(\prod_{k=\nu}^{n-2}\left(\frac{x-\gamma_{k}}{|x-\gamma|} \sqrt{\delta}\right)\right)\left(\frac{2 \sqrt{\delta}}{|x-\gamma|}\right)^{\nu+1}\left\{\cos [n \theta(x)+\eta(x)]+E_{n}(x)\right\} \\
n \geq n_{0}(x)
\end{gathered}
$$

holds (cf. (12), (13), (21), (22)) where

$$
\theta(x):=\arctan \left[\left(\frac{4 \delta}{(x-\gamma)^{2}}-1\right)^{1 / 2}\right]
$$

Setting $x=\gamma+2 \sqrt{\delta} \cos \phi, 0<\phi<\pi, \phi \neq \frac{\pi}{2}$, the latter becomes

$$
\theta(x)=\left\{\begin{array}{lll}
\phi & \text { if } \gamma<x<\gamma+2 \sqrt{\delta} & \text { (i.e., } \left.0<\phi<\frac{\pi}{2}\right) \\
\pi-\phi & \text { if } \gamma-2 \sqrt{\delta}<x<\gamma & \text { (i.e., } \left.\frac{\pi}{2}<\phi<\pi\right)
\end{array}\right.
$$

cf. Example 3.1 concerning Legendre polynomials in [22]. Moreover, the error term in (24), $E_{n}(x)$, can be estimated by

$$
\left|E_{n}(x)\right| \leq \frac{V_{n}(x)}{1-V_{n}(x)}, \quad n \geq n_{0}(x),
$$

where

$$
V_{n}(x):=\left[\delta\left(\delta-\frac{(x-\gamma)^{2}}{4}\right)\right]^{-1 / 2} \sum_{k=n}^{\infty}\left|\frac{\delta_{k}(x-\gamma)^{2}}{\left(x-\gamma_{k}\right)\left(x-\gamma_{k-1}\right)}-\delta\right|,
$$

which follows from (5), (10) and (21). According to (11), in (24), (27), $n_{0}(x)$ is the smallest integer, $n_{0}(x) \geq \nu(x)$, for which $V_{n}(x)<1$.

When the functions $\mathcal{A}(x), \eta(x)$ in (24) can be identified, (24) yields an asymptotic representation with precise error bounds. In any case, (24) provides qualitative information on the asymptotic behavior of the orthogonal polynomials in the BlumenthalNevai subclass characterized by condition (23). The following corollary will be used.

Corollary 2.4. Suppose that the three-term recurrence equation (1) is given with $A_{n}$, $B_{n}$ real valued and

$$
\lim _{n \rightarrow \infty} A_{n}=A \neq 0, \quad \lim _{n \rightarrow \infty} B_{n}=B .
$$

Moreover, suppose that

$$
\sum_{n=\nu+1}^{\infty}\left|\frac{B_{n}}{A_{n} A_{n-1}}-\frac{B}{A^{2}}\right|<\infty
$$

with

$$
\frac{B}{A^{2}}>\frac{1}{4}
$$

Then,

(a) if $0<B<1, Y_{n} \rightarrow 0$ exponentially as $n \rightarrow \infty$;

(b) if $B=1, Y_{n}$ is bounded as $n \rightarrow \infty$, provided that

$$
\left|\sum_{n=\nu}^{\infty}\left(\left|A_{n}\right|-|A|\right)\right|<\infty
$$

(c) if $B>1, Y_{n}$ exhibits exponentially growing amplitude oscillations on suitable subsequences whenever $A_{n}$ has constant sign for $n$ sufficiently large. 
The following corollary, concerning the Blumenthal-Nevai class, can be derived, in part, from Corollary 2.4.

Corollary 2.5. Suppose that a family of orthogonal polynomials in the BlumenthalNevai class (18), (19), satisfying (21) and (23), is given. Then, for each fixed $x \in$ $(\gamma-2 \sqrt{\delta}, \gamma+2 \sqrt{\delta}) \backslash\{\gamma\}$, every solution to (18) is oscillatory (in the classical sense, cf. [21]). Moreover, the following holds:

(a) if $0<\delta<1$, then $P_{n}(x) \rightarrow 0$ exponentially as $n \rightarrow \infty$;

(b) if $\delta=1$, then $P_{n}(x)$ is bounded as $n \rightarrow \infty$, provided that the series $\sum_{n=\nu}^{\infty}\left|\gamma_{n}-\gamma\right|$ converges; and

(c) if $\delta>1$, then $P_{n}(x)$ exhibits exponentially growing amplitude oscillations on suitable subsequences as $n \rightarrow \infty$.

Proof of Corollary 2.4. Noting that (30) and (31) coincide with (15) and (16) with $L=B / A^{2}$, we obtain from (12) (or (17))

$$
Y_{n}=O\left(\prod_{k=\nu}^{n-2}\left(-\frac{A_{k}}{|A|} \sqrt{B}\right)\right), \quad n \geq n_{0},
$$

where $n_{0}$ is defined in (11). From this, parts $(a)$ and $(b)$ of the corollary follow. We only observe that, when $B=1$, condition (32) ensures the convergence of the infinite product $\prod_{k=\nu}^{\infty}\left|A_{k} / A\right|$.

When $B>1$ and $A_{n}$ has constant sign (for $n$ sufficiently large), since $\cos \left(n_{k} \theta+\eta\right)+$ $E_{n_{k}}$ and $\cos \left[n_{h}(\theta+\pi)+\eta\right]+(-1)^{n_{h}} E_{n_{h}}$ alternate in sign and are uniformly bounded away from zero for $k$ and $h$ sufficiently large (cf. Appendix 1), and since the product in (33) increases exponentially as $n \rightarrow \infty, Y_{n_{k}}$ and $Y_{n_{h}}$ exhibit exponentially growing amplitude oscillations (cf. Remark 2.3 and Appendix 1).

Proof of Corollary 2.5. The oscillatory behavior of each solution to (18) follows at once from Remark 2.3, observing that, for every fixed $x \in(\gamma-2 \sqrt{\delta}, \gamma+2 \sqrt{\delta}) \backslash\{\gamma\}$, $A_{n}=A_{n}(x)=\gamma_{n}-x$ does have constant sign for $n$ sufficiently large. The rest of the proof is a mere rephrasing of that of Corollary 2.4.

Remark 2.6. Observe that condition (32), in this case, becomes

$$
\left|\sum_{n=\nu}^{\infty}\left(\left|\gamma_{n}-x\right|-|\gamma-x|\right)\right|<\infty .
$$

In part (b) of Corollary 2.5, however, we gave the stronger condition that $\sum_{n=\nu}^{\infty}\left|\gamma_{n}-\gamma\right|$ converges, which is uniform in $x$. This last condition, together with the convergence of the series $\sum_{n=\nu}^{\infty}\left|\delta_{n}-\delta\right|$, ensures the absolute continuity of the measure associated with (18), cf. [7, Theorem 2.8, p. 104]. Note that such a condition, i.e.,

$$
\sum_{n=\nu}^{\infty}\left(\left|\gamma_{n}-\gamma\right|+\left|\delta_{n}-\delta\right|\right)<\infty
$$

implies our hypothesis (23) which plays a central role in the present theory, as well as in other approaches, e.g. [14]. Indeed, the results given by Corollary 2.4, being essentially qualitative, could be obtained also from [14]. 


\section{Error bounds in the asymptotic representation}

When asymptotic formulae, even merely qualitative (but explicit), already are available, it is possible to identify $\mathcal{A}(x)$ and $\eta(x)$ in (24), thus obtaining an asymptotic representation with a precise error bound. In $\S 3.1$ below, this will be done for the subclass of the Blumenthal-Nevai class satisfying (35). In $\S 3.2$, the case of Jacobi polynomials will be worked out in detail, and in $\S 3.3$ a second-order discrete WKB approximation with error bounds will be developed and applied to the ultraspherical polynomials.

3.1. General results. Without any loss of generality, assume $\gamma=0, \delta=1 / 4$ in (19). Under the hypothesis

$$
\sum^{\infty}\left(\left|\gamma_{k}\right|+\left|\delta_{k}-\frac{1}{4}\right|\right)<\infty
$$

(cf. (35)), Ismail et al. [14] obtained the asymptotic formula

$$
P_{n}(x)=2^{-n}\left(\frac{2\left(\prod_{j=0}^{\infty}\left(4 \delta_{j}\right)\right)}{\pi \sqrt{1-x^{2}} \mu^{\prime}(x)}\right)^{1 / 2} \cos \left(n \theta(x)+\chi(x)-\frac{\pi}{2}+o(1)\right)
$$

where $x \in(-1,1), \theta(x):=\arccos x$, and

$$
\chi(x):=\arccos \left(\frac{Y_{-1}^{(s)}(x+i 0)+Y_{-1}^{(s)}(x-i 0)}{2\left[Y_{-1}^{(s)}(x+i 0) Y_{-1}^{(s)}(x-i 0)\right]^{1 / 2}}\right),
$$

$Y_{n}^{(s)}(z)$ denoting a certain subdominant (or recessive, or minimal) solution to the recurrence equation (18), $z \in \mathbf{C} \backslash[-1,1]$; cf. (3.13) in [14]. In (37), $\mu^{\prime}(x)$ denotes the density of the orthogonality measure, for $x \in(-1,1)$; cf. Remark 2.6. A representation similar to that in (37) is reported in [28].

On the other hand, (24) becomes

$$
\begin{aligned}
P_{n}(x)=2^{-n} & \left(\frac{2}{x}\right)^{\nu+1}(\operatorname{sgn} x)^{n} \mathcal{A}(x)\left(\prod_{k=\nu}^{n-2}\left(1-\frac{\gamma_{k}}{x}\right)\right) \\
& \times\left\{\cos [n \theta(x)+\eta(x)]+E_{n}(x)\right\}, \quad n \geq n_{0}(x), \quad x \neq 0,
\end{aligned}
$$

where

$$
\nu \equiv \nu(x):=\min \left\{k: k \in \mathbf{Z}_{-1}, \gamma_{k} \neq x\right\}
$$

and $n_{0}(x)$ is defined by means of (11). In (39), it is not restrictive to assume $\mathcal{A}(x)>0$, since otherwise we can change $\eta(x)$ into $\eta(x)+\pi$, and $E_{n}(x)$ into $-E_{n}(x)$. Comparing (37) with (39), we get

$$
\begin{aligned}
\left(\frac{2}{x}\right)^{\nu+1} & (\operatorname{sgn} x)^{n} \mathcal{A}(x)\left(\prod_{k=\nu}^{n-2}\left(1-\frac{\gamma_{k}}{x}\right)\right) \cos [n \theta(x)+\eta(x)] \\
& -\left(\frac{2\left(\prod_{j=0}^{\infty}\left(4 \delta_{j}\right)\right)}{\pi \sqrt{1-x^{2}} \mu^{\prime}(x)}\right)^{1 / 2} \cos \left[n \theta(x)+\chi(x)-\frac{\pi}{2}\right]=o(1), \quad n \rightarrow \infty .
\end{aligned}
$$

This can be written, for short, as

$$
a_{n}(x) \cos [n \theta(x)+\eta(x)]-b(x) \cos \left[n \theta(x)+\chi(x)-\frac{\pi}{2}\right]=o(1), \quad n \rightarrow \infty,
$$


with obvious positions. Since the infinite product $\prod_{k=\nu}^{\infty}\left(1-\gamma_{k} / x\right)$ converges (due to (36)), we have $a_{n}(x) \rightarrow a(x)$ as $n \rightarrow \infty$, and thus, from (42),

$$
a(x) \cos \eta(x)=b(x) \cos \left(\chi(x)-\frac{\pi}{2}\right), \quad a(x) \sin \eta(x)=b(x) \sin \left(\chi(x)-\frac{\pi}{2}\right) .
$$

From this, it follows that $a(x)=b(x), \eta(x)=\chi(x)-\pi / 2$, and hence

$$
\mathcal{A}(x)=\left(\frac{x}{2}\right)^{\nu+1}(\operatorname{sgn} x)^{n}\left(\prod_{k=\nu}^{\infty}\left(1-\frac{\gamma_{k}}{x}\right)\right)^{-1}\left(\frac{2\left(\prod_{j=0}^{\infty}\left(4 \delta_{j}\right)\right)}{\pi \sqrt{1-x^{2}} \mu^{\prime}(x)}\right)^{1 / 2} .
$$

The final asymptotic representation for $P_{n}(x)$ is

$$
\begin{aligned}
P_{n}(x)=2^{-n} & \left(\prod_{k=n-1}^{\infty}\left(1-\frac{\gamma_{k}}{x}\right)\right)^{-1}\left(\frac{2\left(\prod_{j=0}^{\infty}\left(4 \delta_{j}\right)\right)}{\pi \sqrt{1-x^{2}} \mu^{\prime}(x)}\right)^{1 / 2} \\
& \times\left\{\sin [n \theta(x)+\chi(x)]+E_{n}(x)\right\}, \quad x \in(-1,1) \backslash\{0\}, \quad n \geq n_{0}(x),
\end{aligned}
$$

where, by (23) and (27),

$$
E_{n}(x)=O\left(V_{n}(x)\right)=O\left(\frac{1}{\sqrt{1-x^{2}}} \sum_{k=n}^{\infty}\left(\left|\frac{\gamma_{k}}{x}\right|+\left|\delta_{k}-\frac{1}{4}\right|\right)\right),
$$

the constant implied by the $O$-symbol being independent of $x$ and $n$.

Remark 3.1. Observe that $n_{0}(x)$ as well as the estimates for $E_{n}(x)$ (valid for $n \geq$ $\left.n_{0}(x)\right)$ can be given uniformly for $x \in[-1+\varepsilon,-\varepsilon] \cup[\varepsilon, 1-\varepsilon]$ for an arbitrary but fixed $\varepsilon, 0<\varepsilon<1 / 2$. The pathological behavior of the representation (45) at the point $x=0$ disappears in the special case $\gamma_{k}=0$ for every $k$ (e.g., for the Jacobi polynomials with $|\alpha|=|\beta|$, see $\S 3.2)$. In this case, in fact, $x=0$ is the only singular point of the transformation $\alpha_{n}(x)$ given by (22), so that we can take $\nu(x)=-1$ for every $x \neq 0$. However, $V_{n}(x)$ in (28) is defined now also at $x=0$, and $E_{n}(x)$ turns out to be continuous in any compact subset $K$ of $(-1,1)$ for $n \geq n_{0}(K) \geq-1$. The latter statement is nontrivial and can be proved on the basis of the uniform convergence of the series (2.11) and (2.16) in [22] defining the error term $\varepsilon_{n}^{+}(x)$ (cf. (7)). Moreover, the estimate for $E_{n}(x)$ can be given uniformly in $K$. Then, the representation (45) holds at $x=0$ when $\gamma_{k} \equiv 0$, and uniformly in every compact subset of $(-1,1)$. In fact, $\mu^{\prime}(x)>0$ is continuous in $(-1,1)$ as well as $\chi(x)$ (cf. Theorem 3 and Corollary 5 in [14]).

3.2. Jacobi polynomials. The case of Jacobi polynomials, which falls in the subclass for which (36) holds, will be worked out in detail in this section. It is less cumbersome to derive this result rather than depending on (45) by comparing (24) with the well-known Darboux-type formula for the monic Jacobi polynomials,

$$
\begin{gathered}
\hat{P}_{n}^{(\alpha, \beta)}(\cos \phi)=f_{n}^{(\alpha, \beta)} P_{n}^{(\alpha, \beta)}(\cos \phi), \quad 0<\phi<\pi, \\
f_{n}^{(\alpha, \beta)}:=\frac{n !}{2^{n}} \frac{\Gamma\left(\frac{\alpha+\beta+1}{2}\right) \Gamma\left(\frac{\alpha+\beta+2}{2}\right)}{\Gamma(\alpha+\beta+1)} \frac{\Gamma(\alpha+\beta+1+n)}{\Gamma\left(\frac{\alpha+\beta+1}{2}+n\right) \Gamma\left(\frac{\alpha+\beta+2}{2}+n\right)},
\end{gathered}
$$


so that

$$
\begin{aligned}
P_{n}^{(\alpha, \beta)}(\cos \phi)=( & \pi n)^{-1 / 2}\left(\sin \frac{\phi}{2}\right)^{-\alpha-1 / 2}\left(\cos \frac{\phi}{2}\right)^{-\beta-1 / 2} \\
& \times \cos \left[\left(n+\frac{\alpha+\beta+1}{2}\right) \phi-\frac{\pi}{2}\left(\alpha+\frac{1}{2}\right)\right]+O\left(n^{-3 / 2}\right),
\end{aligned}
$$

cf. [6, p. 215], [25, (8.21.10), p. 196].

The monic Jacobi polynomials satisfy equation (18) with

$$
\begin{aligned}
& \gamma_{n}=\gamma_{n}^{(\alpha, \beta)}=\frac{\beta^{2}-\alpha^{2}}{(2 n+\alpha+\beta+2)(2 n+\alpha+\beta+4)} \\
& \delta_{n}=\delta_{n}^{(\alpha, \beta)}=\frac{4(n+1)(n+\alpha+1)(n+\beta+1)(n+\alpha+\beta+1)}{(2 n+\alpha+\beta+1)(2 n+\alpha+\beta+2)^{2}(2 n+\alpha+\beta+3)},
\end{aligned}
$$

for $n \geq 1$ (cf. [6, Appendix, p. 220] and [26, p. 271]). One can easily check that they belong to the Blumenthal-Nevai subclass for which (21) and (36) hold. Here, $\gamma=0$ and $\delta=1 / 4$, and hence (21), (23) are satisfied for all $x \in(-1,1) \backslash\{0\}$. The quantity $V_{n}$ in (10) becomes

$$
V_{n}^{(\alpha, \beta)}(x)=\frac{4}{\sqrt{1-x^{2}}} \sum_{k=n}^{\infty}\left|\frac{N_{k}^{(\alpha, \beta)}(x)}{D_{k}^{(\alpha, \beta)}(x)}\right|
$$

where $N_{k}^{(\alpha, \beta)}(x)$ and $D_{k}^{(\alpha, \beta)}(x)$ are polynomials in $k$ of the 4-th and 6-th degree, respectively; the key condition (23) is equivalent to the convergence of the series in (51). The explicit form of $N_{k}^{(\alpha, \beta)}(x)$ is useful in estimating the error term, $E_{n}(x)$, in the asymptotic representation (cf. (27), (28)) and is reported in Appendix 2. Observe that the series in (51) clearly converges for all $x \neq 0$ since $N_{k}^{(\alpha, \beta)}(x) / D_{k}^{(\alpha, \beta)}(x)=O\left(k^{-2}\right)$. The evaluation of $N_{k}^{(\alpha, \beta)}(x)$ is rather cumbersome and has been accomplished by using the symbolic computational device Mathematica [30]. Therefore, for the monic Jacobi polynomials, the asymptotic representation (24) holds, that is

$$
\hat{P}_{n}^{(\alpha, \beta)}(x)=\mathcal{A}(x) \frac{2^{-n+\nu+1}}{|x|^{n}}\left(\prod_{k=\nu}^{n-2}\left(x-\gamma_{k}^{(\alpha, \beta)}\right)\right)\left[\cos (n \theta+\eta(x))+E_{n}(x)\right],
$$

for $n \geq n_{0}(x)$. Here $\theta=\theta(x)$ is defined by (26) with $\gamma=0$ and $\delta=1 / 4$. Moreover, $\nu=\nu(x)=-1$ when $x \neq \gamma_{k}^{(\alpha, \beta)}$ for every $k$. Otherwise, if $x=\gamma_{m}$ for some $m$, $\nu(x)=m+1$.

Now,

$$
\begin{aligned}
& \prod_{k=\nu}^{n-2}\left(x-\gamma_{k}^{(\alpha, \beta)}\right)= \\
& \frac{\prod_{k=\nu}^{n-2}\left\{x\left[4 k^{2}+4 k(\alpha+\beta+3)+(\alpha+\beta+2)(\alpha+\beta+4)\right]+\alpha^{2}-\beta^{2}\right\}}{4^{n-(\nu+1)} \prod_{k=\nu}^{n-2}\left(k+\frac{\alpha+\beta+2}{2}\right) \prod_{k=\nu}^{n-2}\left(k+\frac{\alpha+\beta+4}{2}\right)}
\end{aligned}
$$

and, using formulae (89.1.1), (89.2.1) of [12, p. 482] to express the finite products in (53), we get from the representation (52)

$$
\begin{aligned}
\hat{P}_{n}^{(\alpha, \beta)}(x)= & \mathcal{A}(x) 2^{-n}\left(\frac{2}{x}\right)^{\nu+1}(\operatorname{sgn} x)^{n} \frac{\Gamma\left(\frac{\alpha+\beta+2}{2}+\nu\right) \Gamma\left(\frac{\alpha+\beta+4}{2}+\nu\right)}{\Gamma\left(w^{+}+\nu\right) \Gamma\left(w^{-}+\nu\right)} \\
& \times\left[\cos (n \theta+\eta(x))+E_{n}(x)\right]
\end{aligned}
$$


where we have set, for short,

$$
w^{ \pm}:=\frac{\alpha+\beta+3}{2} \pm \frac{1}{2 x}\left\{x\left[x-\left(\alpha^{2}-\beta^{2}\right)\right]\right\}^{1 / 2} .
$$

(Note, incidentally, that formula (89.2.1) of [12, p. 482] contains a mistake: On the right-hand side, $x^{n}$ must replace $x^{2 n}$.) Observe that $\Gamma\left(w^{+}+\nu\right) \Gamma\left(w^{-}+\nu\right)$ is always real, since $w^{+}$and $w^{-}$are necessarily conjugate to each other whenever they are complex.

Since, by Stirling's formula, $f_{n}^{(\alpha, \beta)}$ in (48) has the asymptotics

$$
f_{n}^{(\alpha, \beta)} \sim \frac{\Gamma\left(\frac{\alpha+\beta+1}{2}\right) \Gamma\left(\frac{\alpha+\beta+2}{2}\right)}{\Gamma(\alpha+\beta+1)} \frac{n^{1 / 2}}{2^{n}} \text { as } n \rightarrow \infty,
$$

by multiplying (47) and (54) by $2^{n}$ and subtracting, we get

$$
\begin{aligned}
& \frac{\Gamma\left(\frac{\alpha+\beta+1}{2}\right) \Gamma\left(\frac{\alpha+\beta+2}{2}\right)}{\sqrt{\pi} \Gamma(\alpha+\beta+1)}\left(\sin \frac{\phi}{2}\right)^{-\alpha-1 / 2}\left(\cos \frac{\phi}{2}\right)^{-\beta-1 / 2} \\
& \times \cos \left[\left(n+\frac{\alpha+\beta+1}{2}\right) \phi-\frac{\pi}{2}\left(\alpha+\frac{1}{2}\right)\right] \\
& -\mathcal{A}(x)\left(\frac{2}{x}\right)^{\nu+1}(\operatorname{sgn} x)^{n} \frac{\Gamma\left(\frac{\alpha+\beta+2}{2}+\nu\right) \Gamma\left(\frac{\alpha+\beta+4}{2}+\nu\right)}{\Gamma\left(w^{+}+\nu\right) \Gamma\left(w^{-}+\nu\right)} \\
& \quad \times \cos [n \theta+\eta(x)]=o(1)
\end{aligned}
$$

as $n \rightarrow \infty$. Considering separately the two cases, $x>0$ and $x<0$, we obtain from (57), (26), recalling that $\eta(x)$ is defined $\bmod \pi$ (cf. [22]),

$$
\begin{aligned}
\mathcal{A}(\cos \phi)= & \frac{1}{\sqrt{\pi}} \frac{\Gamma\left(\frac{\alpha+\beta+1}{2}\right) \Gamma\left(\frac{\alpha+\beta+2}{2}\right)}{\Gamma(\alpha+\beta+1)} \frac{\Gamma\left(w^{+}+\nu\right) \Gamma\left(w^{-}+\nu\right)}{\Gamma\left(\frac{\alpha+\beta}{2}+1+\nu\right) \Gamma\left(\frac{\alpha+\beta}{2}+2+\nu\right)} \\
& \times\left(\frac{1}{2} \cos \phi\right)^{\nu+1}\left(\sin \frac{\phi}{2}\right)^{-\alpha-1 / 2}\left(\cos \frac{\phi}{2}\right)^{-\beta-1 / 2}, \\
\eta(\cos \phi)= & {\left[\left(\frac{\alpha+\beta+1}{2}\right) \phi-\frac{\pi}{2}\left(\alpha+\frac{1}{2}\right)\right] \operatorname{sgn}(\cos \phi) . }
\end{aligned}
$$

Therefore, we get, finally, from (54)

$$
\begin{aligned}
\hat{P}_{n}^{(\alpha, \beta)}(\cos \phi)=\frac{1}{\sqrt{\pi}} & \frac{\Gamma\left(\frac{\alpha+\beta+1}{2}\right) \Gamma\left(\frac{\alpha+\beta+2}{2}\right)}{\Gamma(\alpha+\beta+1)} 2^{-n}\left(\sin \frac{\phi}{2}\right)^{-\alpha-1 / 2}\left(\cos \frac{\phi}{2}\right)^{-\beta-1 / 2} \\
\times & \left\{\cos \left[\left(n+\frac{\alpha+\beta+1}{2}\right) \phi-\frac{\pi}{2}\left(\alpha+\frac{1}{2}\right)\right]\right. \\
& \left.+(\operatorname{sgn}(\cos \phi))^{n} E_{n}(\cos \phi)\right\}
\end{aligned}
$$

for $\phi \in(0, \pi) \backslash\{\pi / 2\}$ and for $n \geq n_{0}(\cos \phi) \geq \nu(\cos \phi)$. Here

$$
\nu(x)=\left\{\begin{array}{ll}
-1 & \text { if } x \neq \gamma_{k}^{(\alpha, \beta)} \\
m+1 & \text { if } x=\gamma_{m}^{(\alpha, \beta)} \text { for all } k,
\end{array} \text { for some } m,\right.
$$

and $n_{0}(x)$ is the smallest index for which $V_{n}^{(\alpha, \beta)}(x)<1, V_{n}^{(\alpha, \beta)}(x)$ being given by $(51)$. 
Consider the case of (monic) ultraspherical polynomials $\hat{P}_{n}^{(\alpha, \alpha)}(x)$. Here Remark 3.1 applies, and after a little algebra we obtain explicitly

$$
V_{n}^{(\alpha, \alpha)}(x)=\frac{4}{\sqrt{1-x^{2}}} \sum_{k=n}^{\infty}\left|\delta_{k}^{(\alpha, \alpha)}-\frac{1}{4}\right|=\frac{\left|1-4 \alpha^{2}\right|}{2 \sqrt{1-x^{2}}(2 n+2 \alpha+1)}, \quad-1<x<1
$$

cf. (28). Correspondingly, we can estimate the absolute error in (60), say $\left|\mathcal{E}_{n}(x)\right|$, as

$$
\begin{aligned}
\left|\mathcal{E}_{n}(x)\right|= & \frac{1}{\sqrt{\pi}} \frac{\Gamma\left(\alpha+\frac{1}{2}\right) \Gamma(\alpha+1)}{\Gamma(2 \alpha+1)} \frac{2^{-n+\alpha+1 / 2}}{\left(1-x^{2}\right)^{\frac{1}{2}\left(\alpha+\frac{1}{2}\right)}}\left|E_{n}(x)\right| \\
\leq & \frac{1}{\sqrt{\pi}} \frac{\Gamma\left(\alpha+\frac{1}{2}\right) \Gamma(\alpha+1)}{\Gamma(2 \alpha+1)} \frac{2^{-n+\alpha+1 / 2}}{\left(1-x^{2}\right)^{\frac{1}{2}\left(\alpha+\frac{1}{2}\right)}} \\
& \times \frac{\left|1-4 \alpha^{2}\right|}{2 \sqrt{1-x^{2}}(2 n+2 \alpha+1)-\left|1-4 \alpha^{2}\right|} .
\end{aligned}
$$

Also, the index $n_{0}(x)$, defined in (11), can be given explicitly as

$$
n_{0}(x)=\left[\left(\alpha+\frac{1}{2}\right)\left(\frac{|1-2 \alpha|}{2 \sqrt{1-x^{2}}}-1\right)\right]+1
$$

where the square brackets denote taking the integral part.

Going back to the usual (non-monic) ultraspherical polynomials, for the purpose of comparison with known formulae, one discovers that our representation (60) for $\beta=\alpha$ leads to formula (8.21.14) of [25, p. 197] at the lowest order, which is the generalization of the Darboux formula for Legendre polynomials to the ultraspherical case. In doing that, both the factor $f_{n}^{(\alpha, \alpha)}$ in (48) and the appropriate normalization of ultraspherical polynomials (the factor between $P_{n}^{(\alpha, \alpha)}(x)$ and $P_{n}^{(\lambda)}(x)$, cf. (4.7.1) of $[25$, p. 80]) have been taken into account, and the duplication formula for the gamma function has been used. Moreover, in (8.21.14) of [25, p. 197] with $p=1$, i.e.,

$$
P_{n}^{(\lambda)}(\cos \phi)=\frac{2}{\Gamma(\lambda)} \frac{\Gamma(\lambda+n)}{n !} \frac{1}{(2 \sin \phi)^{\lambda}} \cos \left[(n+\lambda) \phi-\lambda \frac{\pi}{2}\right]+\Xi_{n}^{(\lambda)}, \quad \phi \in(0, \pi),
$$

the absolute error, $\left|\Xi_{n}^{(\lambda)}\right|$, can be precisely estimated as

$$
\begin{aligned}
\left|\Xi_{n}^{(\lambda)}\right| & =\frac{2}{n !}\left|\frac{\Gamma(\lambda+n)}{\Gamma(\lambda)}\right|\left|\mathcal{E}_{n}\right| \\
& \leq 2 \frac{|\lambda(1-\lambda)|}{|\Gamma(\lambda)|} \frac{|\Gamma(\lambda+n)|}{n !} \frac{1}{(2 \sin \phi)^{\lambda}} \frac{1}{(n+\lambda) \sin \phi-|\lambda(1-\lambda)|}
\end{aligned}
$$

cf. (63). Obviously, this is useful for computational purposes for large $n$ only when $\lambda<2$ since $\Xi_{n}^{(\lambda)}$ is infinitesimal as $n \rightarrow \infty$ for such values of $\lambda$.

As for the comparison with the available results in the literature for general Jacobi polynomials, we observe what follows:

Remark 3.2. When $\alpha>-1 / 2, \alpha-\beta>-4, \alpha+\beta \geq-1$, and $x \in[0,1]$, an asymptotic formula with a bound can be found for $P_{n}^{(\alpha, \beta)}(x)$ in [9]. By the reflection formula, $P_{n}^{(\alpha, \beta)}(x)=(-1)^{n} P_{n}^{(\beta, \alpha)}(-x),[25,(4.1 .3)]$, one then can obtain a representation (with a bound) valid in $[-1,0]$, at the price of restricting $\alpha, \beta$ to $\beta>-1 / 2, \beta-\alpha>-4$, and $\alpha+\beta \geq-1$. Consequently, a representation (with a bound) valid on the whole interval $[-1,1]$ holds when both restrictions above are valid, namely $\alpha, \beta>-1 / 2,|\alpha-\beta|<4$, 
and $\alpha+\beta \geq-1$. Note that such conditions reduce to $\lambda>0$ for the ultraspherical polynomials. In [2], similar results have been obtained under the stronger limitations $-1 / 2<\alpha, \beta<1 / 2$; Hahn [11] gave different asymptotic expansions with bounds, under the same restrictions. In [2] the cases $\alpha= \pm 1 / 2$ and/or $\beta= \pm 1 / 2$ also were included.

Even though, when these formulae can be applied they perform better than ours, our estimates hold for any choice of the parameters (in particular, for $\alpha$ and/or $\beta \leq$ $-1 / 2)$, and for $x \in(-1,1) \backslash\{0\}$. In the ultraspherical case, our results hold on the whole interval $(-1,1)$, even for $\lambda<0$. It seems, therefore, that the present results contribute to the completion of the asymptotic theory for the Jacobi polynomials in the orthogonality interval.

In view of Remark 3.2, it is worthwhile to give more explicit estimates for $V_{n}^{(\alpha, \beta)}(x)$ in (51), which might be useful also for computational purposes. After a little algebra, we obtain (see Appendix 2 and (51))

$$
\begin{aligned}
\left|\frac{N_{k}^{(\alpha, \beta)}(x)}{D_{k}^{(\alpha, \beta)}(x)}\right|= & \frac{k^{4}\left|\sum_{s=0}^{4} a_{s}(x, \alpha, \beta) k^{s-4}\right|}{4 \sqrt{1-x^{2}}\left|x-\gamma_{k}^{(\alpha, \beta)}\right|\left|x-\gamma_{k-1}^{(\alpha, \beta)}\right|(2 k+\alpha+\beta+2)} \\
& \times \frac{1}{\Pi_{j=0}^{4}(2 k+\alpha+\beta+j)}, \quad k \geq \nu(x) .
\end{aligned}
$$

Noting that, for $k \geq n$ and $\alpha+\beta>-2,2 k+\alpha+\beta \geq 2 k(1-1 / n), 2 k+\alpha+\beta+1 \geq$ $2 k(1-1 / 2 n)$, and $2 k+\alpha+\beta+j>2 k$ for $j=2,3,4$, we get

$$
\begin{aligned}
& V_{n}^{(\alpha, \beta)}(x) \leq \\
& \quad \frac{\left(\sum_{i=0}^{4}\left|a_{i 2}(\alpha, \beta)\right| n^{i-4}\right) x^{2}+\left(\sum_{i=0}^{4}\left|a_{i 1}(\alpha, \beta)\right| n^{i-4}\right)|x|+\sum_{i=0}^{4}\left|a_{i 0}(\alpha, \beta)\right| n^{i-4}}{64 \sqrt{1-x^{2}}(1-1 / n)(1-1 / 2 n)} \\
& \quad \times \sum_{k=n}^{\infty} \frac{1}{k^{2}\left|x-\gamma_{k}^{(\alpha, \beta)}\right|\left|x-\gamma_{k-1}^{(\alpha, \beta)}\right|}, \quad n \geq \max \{2, \nu(x)\} .
\end{aligned}
$$

To display dependence on $n$, we write the final pointwise estimate for $x \in(-1,1) \backslash\{0\}$ as

$$
V_{n}^{(\alpha, \beta)}(x) \leq \frac{\psi_{n}^{(\alpha, \beta)}(x)}{n \sqrt{1-x^{2}}}=O\left(\frac{1}{n}\right)
$$

where

$$
\begin{aligned}
\psi_{n}^{(\alpha, \beta)}(x):= & \frac{1+1 / 2 n+1 / 6 n^{2}}{64(1-1 / n)(1-1 / 2 n)}\left[\left(\sum_{i=0}^{4}\left|a_{i 2}(\alpha, \beta)\right| n^{i-4}\right) x^{2}\right. \\
& \left.+\left(\sum_{i=0}^{4}\left|a_{i 1}(\alpha, \beta)\right| n^{i-4}\right)|x|+\sum_{i=0}^{4}\left|a_{i 0}(\alpha, \beta)\right| n^{i-4}\right] \xi_{n}^{(\alpha, \beta)}(x)
\end{aligned}
$$

and

$$
\xi_{n}^{(\alpha, \beta)}(x):= \begin{cases}\left(x-\gamma_{n}^{(\alpha, \beta)}\right)^{-1}\left(x-\gamma_{n-1}^{(\alpha, \beta)}\right)^{-1} & \text { if }|\beta| \geq|\alpha|, x>\gamma_{n-1}^{(\alpha, \beta)}, \\ & \text { or if }|\beta|<|\alpha|, x<\gamma_{n-1}^{(\alpha, \beta)}, \\ x^{-2} & \text { if }|\beta| \geq|\alpha|, x<0, \\ & \text { or if }|\beta|<|\alpha|, x>0,\end{cases}
$$


is an estimate of $\left|x-\gamma_{k}^{(\alpha, \beta)}\right|^{-1}\left|x-\gamma_{k-1}^{(\alpha, \beta)}\right|^{-1}$ for $k \geq n \geq 2$. Note that

$$
\begin{aligned}
\lim _{n \rightarrow \infty} \psi_{n}^{(\alpha, \beta)}(x) & =\frac{1}{64}\left(\left|a_{42}(\alpha, \beta)\right|+\frac{\left|a_{41}(\alpha, \beta)\right|}{|x|}\right) \\
& =\frac{1}{4}\left(\left|1-2\left(\alpha^{2}-\beta^{2}\right)\right|+\frac{2\left|\alpha^{2}-\beta^{2}\right|}{|x|}\right) .
\end{aligned}
$$

Clearly, the restrictions shown in the first relation of (71) imply that, for each fixed $x, n$ should be chosen suitably large, in view of the fact that $\gamma_{n}^{(\alpha, \beta)} \sim$ const $/ n^{2}$ as $n \rightarrow \infty$, and the sign of $\gamma_{n}^{(\alpha, \beta)}$ is the same as $|\beta|-|\alpha|$. Recall that an additional restriction on $n$ could be required in (60) (cf. (27)) to ensure that $V_{n}^{(\alpha, \beta)}(x)<1$.

Uniform estimates also can be derived starting from (69)-(71). Let $\varepsilon>0,0<\eta<$ $1-\varepsilon$. Then, when $x \in[\eta, 1-\varepsilon]$,

$$
V_{n}^{(\alpha, \beta)}(x) \leq \frac{\psi_{n}^{(\alpha, \beta)}(\eta)}{n \sqrt{\varepsilon(2-\varepsilon)}}, \quad \forall n \geq \max \left\{2, \mu_{1}\right\}, \quad \mu_{1}:=\min \left\{n: \gamma_{n-1}^{(\alpha, \beta)}<\eta\right\} ;
$$

when $x \in[-1+\varepsilon,-\eta]$,

$$
V_{n}^{(\alpha, \beta)}(x) \leq \frac{\psi_{n}^{(\alpha, \beta)}(-\eta)}{n \sqrt{\varepsilon(2-\varepsilon)}}, \quad \forall n \geq \max \left\{2, \mu_{2}\right\}, \quad \mu_{2}:=\min \left\{n: \gamma_{n-1}^{(\alpha, \beta)}>-\eta\right\} .
$$

In order to estimate the error term $E_{n}$ in (60) uniformly through (27), (73) and (74), $n$ should be chosen so large that $V_{n}^{(\alpha, \beta)}(x)<1$ uniformly in $x$ in the appropriate interval.

We observe in closing that our estimates can be generalized by minor modifications in (68) to the case of all the real values of $\alpha$ and $\beta$ not included in (68) (i.e., $\alpha+\beta \leq-2$ ). In fact, our theory as well as the basic formula (8.21.8) in Szegö's book are free from restrictions on $\alpha$ and $\beta$. When $\alpha+\beta \leq-2$, it suffices to replace $(1-1 / n)(1-1 / 2 n)$ in the right-hand side of (68) with $(1-|\alpha+\beta| / 2 n)^{6}$, since $2 k+\alpha+\beta+j \geq 2 k(1-|\alpha+\beta| / 2 n)$, under the additional restriction $n>|\alpha+\beta| / 2$.

3.3. Second-order discrete WKB approximations. It is possible to improve the discrete Liouville-Green (WKB) theory developed by the authors, obtaining higherorder approximations, by splitting $\varepsilon_{n}^{ \pm}$as $\varepsilon_{n}^{ \pm}=\xi_{n}^{ \pm}+e_{n}^{ \pm}$, so that

$$
y_{n}^{ \pm}=\rho^{n}\left(1+\xi_{n}^{ \pm}+e_{n}^{ \pm}\right) e^{ \pm i n \theta} \text {, }
$$

cf. (7) and (13), with

$$
\xi_{n}^{ \pm}=O\left(V_{n}\right), \quad e_{n}^{ \pm}=o\left(V_{n}\right), \quad n \rightarrow \infty,
$$

where $\xi_{n}^{ \pm}$is explicitly representable and $e_{n}^{ \pm}$can be estimated. In fact, in view of the (discrete) Liouville-Neumann expansion of the error term in (7) [22, 23], we have

$$
\varepsilon_{n}^{ \pm}=h_{s}^{ \pm}(n)+r_{s}^{ \pm}(n), \quad\left|r_{s}^{ \pm}(n)\right| \leq \frac{\left(V_{n}\right)^{s+1}}{1-V_{n}}, \quad s=1,2, \ldots, \quad n \geq n_{0},
$$

where $h_{s}^{ \pm}(n)=O\left(V_{n}\right)$ is recursively defined by

$$
\begin{gathered}
h_{0}^{ \pm}(n) \equiv 0, \quad h_{s+1}^{ \pm}(n)=\frac{1}{2 \lambda^{ \pm}\left(\lambda^{ \pm}-1\right)} \sum_{j=n}^{\infty}\left[1-\left(\frac{\lambda^{ \pm}}{\lambda^{\mp}}\right)^{j-n+1}\right] g_{j}\left(1+h_{s}^{ \pm}(j)\right), \\
s=0,1,2, \ldots
\end{gathered}
$$


In what follows, $\xi_{n}^{ \pm}$in (75) will be identified with $h_{s}^{ \pm}(n)$ or with its "dominant part" with respect to $V_{n}$ (as $\left.n \rightarrow \infty\right)$. This approach can be pursued for $s=1$ (secondorder approximation) exploiting suitable assumptions on the asymptotic behavior of the sequence $g_{n}$, while the case $s>1$ seems to be much more difficult to treat explicitly. Recall that, when $g_{n} \in \mathbf{R}, \varepsilon_{n}^{+}$and $\varepsilon_{n}^{-}$as well as $h_{s}^{+}(n)$ and $h_{s}^{-}(n)$ are conjugate; in the applications below, $\xi_{n}^{+}$and $\xi_{n}^{-}$also turn out to be conjugate, and so do $e_{n}^{+}$and $e_{n}^{-}$.

Considering the general real solution to (1) under the assumptions (15) and (16),

$$
Y_{n}=\alpha_{n} \rho^{n}\left[c_{1} e^{i n \theta}\left(1+\xi_{n}^{+}+e_{n}^{+}\right)+c_{2} e^{-i n \theta}\left(1+\xi_{n}^{-}+e_{n}^{-}\right)\right]
$$

where $c_{2}=\overline{c_{1}}$, we obtain, after simple manipulations,

$$
Y_{n}=\mathcal{A} \alpha_{n} \rho^{n}\left[\cos (n \theta+\eta)+\left|\xi_{n}^{+}\right| \cos \left(n \theta+\eta+\arg \xi_{n}^{+}\right)+\tilde{E}_{n}\right]
$$

where

$$
\tilde{E}_{n}:=\left|e_{n}^{+}\right| \cos \left(n \theta+\eta+\arg e_{n}^{+}\right) .
$$

The latter shows, in particular, that $\tilde{E}_{n}=o\left(V_{n}\right)$ in view of (76).

As a first example, suppose that in (5)

$$
g_{n}=c n^{-p}+O\left(n^{-q}\right), \quad c \in \mathbf{R}, \quad n \geq-1,
$$

where $q>p>1$. From (78) with $s=0$ and (82), we obtain

$$
\begin{aligned}
h_{1}^{+}(n)= & \frac{1}{2 \lambda^{+}\left(\lambda^{+}-1\right)}\left\{c \sum_{j=n}^{\infty} j^{-p}-c\left(\frac{\lambda^{+}}{\lambda^{-}}\right)^{1-n} \sum_{j=n}^{\infty}\left(\frac{\lambda^{+}}{\lambda^{-}}\right)^{j} j^{-p}\right. \\
& \left.+\sum_{j=n}^{\infty} O\left(j^{-q}\right)-\left(\frac{\lambda^{+}}{\lambda^{-}}\right)^{1-n} \sum_{j=n}^{\infty}\left(\frac{\lambda^{+}}{\lambda^{-}}\right)^{j} O\left(j^{-q}\right)\right\} .
\end{aligned}
$$

Using well-known asymptotic results for the remainder of the power series defining the Riemann $\zeta$-function (for the first and the third terms inside parentheses), and of the power series defining the generalized "polylogarithm" on the boundary of the unit disc (for the second and the fourth terms, since $\left|\lambda^{+} / \lambda^{-}\right|=1, \lambda^{+} / \lambda^{-} \neq 1$ ) [20, Ch.8], we get

$$
h_{1}^{+}(n)=\frac{1}{2 \lambda^{+}\left(\lambda^{+}-1\right)} \frac{c}{p-1} n^{1-p}+O\left(n^{-\min \{p, q-1\}}\right),
$$

and thus, by (77),

$$
\varepsilon_{n}^{+}=\frac{1}{2 \lambda^{+}\left(\lambda^{+}-1\right)} \frac{c}{p-1} n^{1-p}+O\left(n^{-\min \{p, q-1,2 p-2\}}\right) .
$$

Finally, by (80),

$$
\begin{aligned}
Y_{n}=\mathcal{A} \alpha_{n} \rho^{n}\left(\cos (n \theta+\eta)+\frac{c}{2(p-1) \sqrt{a(a+1)}}\right. \\
\left.\times n^{1-p} \sin [(n-1) \theta+\eta]+\tilde{E_{n}}\right), \quad n \geq n_{0},
\end{aligned}
$$

with $n_{0}$ defined in (11), and

$$
\tilde{E}_{n}=O\left(n^{-\min \{p, q-1,2 p-2\}}\right) .
$$

We stress that all $O$-terms in the formulae above could be explicitly estimated, provided that an estimate for the constant implied by the $O$-symbol in (82) is known.

As an application to the Blumenthal-Nevai polynomials, consider for simplicity the subclass $\gamma_{k} \equiv 0$ and $\delta_{k}=1 / 4+c n^{-p}+O\left(n^{-q}\right), q>p>1$ (cf. Remark 3.1); such a 
class satisfies (36) and includes, in particular, the ultraspherical polynomials $(p=2$, $q=3$ ). Therefore, the representation (45) can be improved, in this case, to

$$
\begin{aligned}
P_{n}(x)=2^{-n} & \left(\frac{2\left(\prod_{j=0}^{\infty}\left(4 \delta_{j}\right)\right)}{\pi \sqrt{1-x^{2}} \mu^{\prime}(x)}\right)^{1 / 2}\{\sin [n \theta(x)+\chi(x)] \\
& -\frac{c}{2(p-1)} \frac{x^{2}}{\sqrt{1-x^{2}}} n^{1-p} \cos [(n-1) \theta(x)+\chi(x)] \\
& \left.+O\left(n^{-\min \{p, q-1,2 p-2\}}\right)\right\}, \quad x \in(-1,1), \quad n \geq n_{0}(x) .
\end{aligned}
$$

Even though the capital $O$-term in (88) could be estimated (as pointed out above), in the case of ultraspherical polynomials it is more convenient to choose a different splitting of the first-order error term:

$$
\begin{aligned}
& \xi_{n}^{+}:=\frac{1}{2 \lambda^{+}\left(\lambda^{+}-1\right)} \sum_{j=n}^{\infty} g_{j}, \quad g_{j}=\frac{4}{x^{2}}\left(\delta_{j}-\frac{1}{4}\right) \\
& e_{n}^{+}:=-\frac{1}{2 \lambda^{+}\left(\lambda^{+}-1\right)} \sum_{j=n}^{\infty} g_{j}\left(\frac{\lambda^{+}}{\lambda^{-}}\right)^{j-n+1}+r_{1}^{+}(n)
\end{aligned}
$$

(cf. (77), (78)) where

$$
\lambda^{+}=1+i \sqrt{\frac{1}{x^{2}}-1}, \quad \delta_{j} \equiv \delta_{j}^{(\alpha, \alpha)}=\frac{(j+1)(j+2 \alpha+1)}{(2 j+2 \alpha+1)(2 j+2 \alpha+3)}
$$

(cf. (50)). Summing up the telescopic series in (89) explicitly, it follows that

$$
\xi_{n}^{+}=-\frac{i}{4} \frac{1-4 \alpha^{2}}{\sqrt{1-x^{2}}\left(|x|+i \sqrt{1-x^{2}}\right)} \frac{1}{2 n+2 \alpha+1}
$$

and

$$
\left|\xi_{n}^{+}\right|=\frac{V_{n}}{2}, \quad \arg \xi_{n}^{+}=-\theta-\frac{\pi}{2} \operatorname{sgn}\left(1-4 \alpha^{2}\right)
$$

(see (62) for the definition of $\left.V_{n} \equiv V_{n}^{(\alpha, \alpha)}\right)$. An estimate of $e_{n}^{+}$, defined in (90), is obtained by summing by parts and using the telescopic property of $\sum\left(g_{j+1}-g_{j}\right)$, after rather long but simple calculations. The result is

$$
\begin{aligned}
\left|e_{n}^{+}\right| \leq & \frac{\left|1-4 \alpha^{2}\right|+3|2 \alpha+1|(2 \alpha+3)}{2\left(1-x^{2}\right)(2 n+2 \alpha+1)(2 n+2 \alpha+3)} \\
& +\frac{\left(1-4 \alpha^{2}\right)^{2}}{2 \sqrt{1-x^{2}}(2 n+2 \alpha+1)} \frac{1}{2 \sqrt{1-x^{2}}(2 n+2 \alpha+1)-\left|1-4 \alpha^{2}\right|}, \quad n \geq n_{0}(x) .
\end{aligned}
$$

Finally, we have the second-order asymptotic representation for the non-monic ultraspherical polynomials, $P_{n}^{(\lambda)}(\cos \phi), \alpha=\lambda-1 / 2$,

$$
\begin{aligned}
P_{n}^{(\lambda)}(\cos \phi)= & \frac{2}{\Gamma(\lambda)} \frac{\Gamma(\lambda+n)}{n !} \frac{1}{(2 \sin \phi)^{\lambda}}\left\{\cos \left[(n+\lambda) \phi-\lambda \frac{\pi}{2}\right]\right. \\
& +\frac{\lambda(1-\lambda)}{2(n+\lambda) \sin \phi} \operatorname{sgn}(\cos \phi) \cos \left[(n+\lambda-1) \phi-\lambda \frac{\pi}{2}\right] \\
& \left.+(\operatorname{sgn}(\cos \phi))^{n} \tilde{E}_{n}\right\}, \quad \phi \in(0, \pi),
\end{aligned}
$$


$n \geq n_{0}(\cos \phi)$, where

$$
\begin{aligned}
\left|\tilde{E}_{n}\right| \leq\left|e_{n}^{+}\right| \leq & \frac{|\lambda|(|1-\lambda|+3(1+\lambda))}{2(n+\lambda)(n+\lambda+1) \sin ^{2} \phi} \\
& +\frac{\lambda^{2}(1-\lambda)^{2}}{(n+\lambda) \sin \phi} \frac{1}{(n+\lambda) \sin \phi-|\lambda(1-\lambda)|} .
\end{aligned}
$$

In closing, we stress that the representation (95) along with the bound (96) hold for all values of $\lambda>-1$, including the negative values, which case seems to be missing in the literature.

\section{Appendix 1}

As is known, a sequence $Y_{n}$ is called oscillatory if, for every $N \in \mathbf{N}$, there is an index $m>N$ such that $Y_{m} Y_{m+1} \leq 0$, cf. [21]. Therefore, it suffices to show that the sequence in (12) is oscillatory on a suitable subsequence, say $\left\{n_{k}\right\}$, whenever $A_{n}$ has constant sign for $n$ sufficiently large.

Suppose first that $A_{n}<0$ for $n \geq \mu \geq n_{0}$, for some $\mu \in \mathbf{N}$. Then $\alpha_{n}$ has a constant sign for $n \geq \mu$, and the oscillatory character of $Y_{n}$ and that of $\cos (n \theta+\eta)+E_{n}$ is the same. We can show first that $\cos (n \theta+\eta)$ alternates in sign on a suitable subsequence. In fact, for each fixed value of $\theta$ and $\eta$, with $0<\theta<\pi / 2$ (cf. (13)), there is at least one integer $n_{k}$ such that

$$
\frac{2 k \pi-\eta-\pi / 4}{\theta}<n_{k}<\frac{2 k \pi-\eta+\pi / 4}{\theta}
$$

for each $k \in \mathbf{N}$, since the length of such an interval is $L=\pi / 2 \theta>1$. Moreover, clearly, any two consecutive intervals of this type do not overlap, so that choosing, e.g., the smallest value satisfying (97) in each interval, we get a subsequence $\left\{n_{k}\right\}$ on which $\cos \left(n_{k} \theta+\eta\right)>1 / \sqrt{2}$.

Similarly, replacing $2 k \pi$ in (97) with $(2 h+1) \pi$, we get a subsequence $\left\{n_{h}\right\}$ such that $\cos \left(n_{h} \theta+\eta\right)<-1 / \sqrt{2}$. From these two subsequences another can be constructed, which we rename $\left\{n_{k}\right\}$, such that $\cos \left(n_{k} \theta+\eta\right)$ alternates in sign and $\left|\cos \left(n_{k} \theta+\eta\right)\right|>$ $1 / \sqrt{2}$. Therefore, for $k$ sufficiently large, $\cos \left(n_{k} \theta+\eta\right)+E_{n_{k}}$ also alternates in sign and $\left|\cos \left(n_{k} \theta+\eta\right)+E_{n_{k}}\right| \geq c>0$, for some constant $c$.

If, finally, $A_{n}>0$ for $n \geq \mu \geq n_{0}$, for some $\mu \in \mathbf{N}$, the factor $(-1)^{n}$ deriving from the transformation $\alpha_{n}$ can be absorbed in the $\cos$ term by replacing $\theta$ with $\theta+\pi$ (and $E_{n}$ with $\left.(-1)^{n} E_{n}\right)$. The same conclusion then follows.

\section{Appendix 2}

The polynomials $N_{n}^{(\alpha, \beta)}(x), D_{n}^{(\alpha, \beta)}(x)$ in $n$ appearing in (51) (which are also polynomials in $x, \alpha, \beta)$, can be evaluated with the help of symbolic computation, more precisely by using Mathematica [30]. In view of (67), we only need to consider

$$
N_{n}^{(\alpha, \beta)}(x)=\sum_{k=0}^{4} a_{k}(x, \alpha, \beta) n^{k}
$$

where

$$
a_{k}(x, \alpha, \beta):=\sum_{j=0}^{2} a_{k j}(\alpha, \beta) x^{j}, \quad k=0, \ldots, 4
$$




$$
\begin{aligned}
& a_{00}(\alpha, \beta)=-3 \alpha^{4}-4 \alpha^{5}-\alpha^{6}-4 \alpha^{4} \beta-2 \alpha^{5} \beta+6 \alpha^{2} \beta^{2}+8 \alpha^{3} \beta^{2}+\alpha^{4} \beta^{2} \\
& +8 \alpha^{2} \beta^{3}+4 \alpha^{3} \beta^{3}-3 \beta^{4}-4 \alpha \beta^{4}+\alpha^{2} \beta^{4}-4 \beta^{5}-2 \alpha \beta^{5}-\beta^{6}, \\
& a_{01}(\alpha, \beta)=-24 \alpha^{2}-56 \alpha^{3}-46 \alpha^{4}-16 \alpha^{5}-2 \alpha^{6}-56 \alpha^{2} \beta-92 \alpha^{3} \beta-48 \alpha^{4} \beta \\
& -8 \alpha^{5} \beta+24 \beta^{2}+56 \alpha \beta^{2}-32 \alpha^{3} \beta^{2}-10 \alpha^{4} \beta^{2}+56 \beta^{3}+92 \alpha \beta^{3} \\
& +32 \alpha^{2} \beta^{3}+46 \beta^{4}+48 \alpha \beta^{4}+10 \alpha^{2} \beta^{4}+16 \beta^{5}+8 \alpha \beta^{5}+2 \beta^{6}, \\
& a_{02}(\alpha, \beta)=16 \alpha+20 \alpha^{2}-24 \alpha^{3}-39 \alpha^{4}-12 \alpha^{5}-\alpha^{6}+16 \beta+40 \alpha \beta-8 \alpha^{2} \beta \\
& -76 \alpha^{3} \beta-44 \alpha^{4} \beta-6 \alpha^{5} \beta+20 \beta^{2}-8 \alpha \beta^{2}-74 \alpha^{2} \beta^{2}-72 \alpha^{3} \beta^{2} \\
& -15 \alpha^{4} \beta^{2}-24 \beta^{3}-76 \alpha \beta^{3}-72 \alpha^{2} \beta^{3}-20 \alpha^{3} \beta^{3}-39 \beta^{4} \\
& -44 \alpha \beta^{4}-15 \alpha^{2} \beta^{4}-12 \beta^{5}-6 \alpha \beta^{5}-\beta^{6} \text {, } \\
& a_{10}(\alpha, \beta)=-8 \alpha^{4}-4 \alpha^{5}-4 \alpha^{4} \beta+16 \alpha^{2} \beta^{2}+8 \alpha^{3} \beta^{2}+8 \alpha^{2} \beta^{3}-8 \beta^{4}-4 \alpha \beta^{4}-4 \beta^{5} \text {, } \\
& a_{11}(\alpha, \beta)=-112 \alpha^{2}-184 \alpha^{3}-96 \alpha^{4}-16 \alpha^{5}-184 \alpha^{2} \beta-192 \alpha^{3} \beta-48 \alpha^{4} \beta \\
& +112 \beta^{2}+184 \alpha \beta^{2}-32 \alpha^{3} \beta^{2}+184 \beta^{3}+192 \alpha \beta^{3}+32 \alpha^{2} \beta^{3}+96 \beta^{4} \\
& +48 \alpha \beta^{4}+16 \beta^{5} \\
& a_{12}(\alpha, \beta)=32+80 \alpha-16 \alpha^{2}-152 \alpha^{3}-88 \alpha^{4}-12 \alpha^{5}+80 \beta+96 \alpha \beta-136 \alpha^{2} \beta \\
& -192 \alpha^{3} \beta-44 \alpha^{4} \beta-16 \beta^{2}-136 \alpha \beta^{2}-208 \alpha^{2} \beta^{2}-72 \alpha^{3} \beta^{2}-152 \beta^{3} \\
& -192 \alpha \beta^{3}-72 \alpha^{2} \beta^{3}-88 \beta^{4}-44 \alpha \beta^{4}-12 \beta^{5} \text {, }
\end{aligned}
$$

$$
\begin{aligned}
a_{20}(\alpha, \beta)=- & 4 \alpha^{4}+8 \alpha^{2} \beta^{2}-4 \beta^{4} \\
a_{21}(\alpha, \beta)=- & 184 \alpha^{2}-192 \alpha^{3}-48 \alpha^{4}-192 \alpha^{2} \beta-96 \alpha^{3} \beta+184 \beta^{2}+192 \alpha \beta^{2} \\
& +192 \beta^{3}+96 \alpha \beta^{3}+48 \beta^{4} \\
a_{22}(\alpha, \beta)=80 & +96 \alpha-136 \alpha^{2}-192 \alpha^{3}-44 \alpha^{4}+96 \beta+48 \alpha \beta-192 \alpha^{2} \beta \\
& \quad-96 \alpha^{3} \beta-136 \beta^{2}-192 \alpha \beta^{2}-104 \alpha^{2} \beta^{2}-192 \beta^{3}-96 \alpha \beta^{3}-44 \beta^{4},
\end{aligned}
$$

$$
\begin{aligned}
& a_{30}(\alpha, \beta)=0, \quad a_{31}(\alpha, \beta)=-128 \alpha^{2}-64 \alpha^{3}-64 \alpha^{2} \beta+128 \beta^{2}+64 \alpha \beta^{2}+64 \beta^{3} \\
& a_{32}(\alpha, \beta)=64+32 \alpha-128 \alpha^{2}-64 \alpha^{3}+32 \beta-64 \alpha^{2} \beta-128 \beta^{2}-64 \alpha \beta^{2}-64 \beta^{3}
\end{aligned}
$$

$$
a_{40}(\alpha, \beta)=0, \quad a_{41}(\alpha, \beta)=-32 \alpha^{2}+32 \beta^{2}, \quad a_{42}(\alpha, \beta)=16-32 \alpha^{2}-32 \beta^{2}
$$

Acknowledgement. This work has been supported, in part, by MURST Numerical Analysis " $40 \%$ "-funds, MURST Mathematical Analysis "60\%"-funds, GNFM-CNR, and GNIM-CNR.

\section{References}

1. R.P. Agarwal, Difference Equations and Inequalities, Marcel Dekker, New York, 1991.

2. P. Baratella and L. Gatteschi, The bounds for the error term of an asymptotic approximation of Jacobi polynomials. In: Orthogonal Polynomials and Their Applications (Eds. M. Alfaro, J.S. Dehesa, F. Marcellan, J.L. Rubio de Francia, and J. Vinuesa), Lecture Notes in Mathematics, vol.1329, Springer-Verlag, Berlin, 1988, pp. 203-221. 
3. O. Blumenthal, Über die Entwicklung einer willkürlichen Funktion nach den Nennern des Kettenbruches für $\int_{-\infty}^{0}[\phi(\xi) /(z-\xi)] d \xi$, Dissertation, Göttingen, 1898.

4. P.A. Braun, WKB method for three-term recurrence relations and quasi energies of an anharmonic oscillator, Theor. Math. Phys. 37 (1978), 1070-1081.

5. K.M. Case, Orthogonal polynomials revisited. In: Theory and Application of Special Functions (Ed. R.A. Askey), Academic Press, New York, 1975.

6. T.S. Chihara, An Introduction to Orthogonal Polynomials, Gordon and Breach, New York, 1978.

7. - The three term recurrence relation and spectral properties of orthogonal polynomials. In: Orthogonal Polynomials: Theory and Practice (Ed. P.G. Nevai), NATO ASI Series, Kluwer, Dordrecht, 1990, pp. 99-114.

8. R.B. Dingle and G.J. Morgan, WKB methods for difference equations, Appl. Sci. Res. 18 (1967), 221-244.

9. C.L. Frenzen and R. Wong, A uniform asymptotic expansion of the Jacobi polynomials with error bounds, Can. J. Math. 37 (1985), 979-1007.

10. J. Geronimo and D. Smith, WKB (Liouville-Green) analysis of second order difference equations and applications, J. Approx. Theory 69 (1992), 269-301.

11. E. Hahn, Asymptotik bei Jacobi-Polynomen und Jacobi-Funktionen, Math. Zeit. 171 (1980), 201-226.

12. E.R. Hansen, A Table of Series and Products, Prentice-Hall, Englewood Cliffs, N.J., 1975.

13. W.G. Kelley and A.G. Peterson, Difference Equations, Academic Press, San Diego, 1991.

14. M.E.H. Ismail, D.R. Masson, and E.B. Saff, A minimal solution approach to polynomial asymptotics. In: Orthogonal Polynomials and Their Applications (Eds. C. Brezinski, L. Gori, and A. Ronveaux), IMACS, Baltzer, Basel, 1991, pp. 299-303.

15. V. Lakshmikantham and D. Trigiante, Theory of Difference Equations: Numerical Methods and Applications, Academic Press, San Diego, 1988.

16. P.G. Nevai, Orthogonal Polynomials, Mem. Amer. Math. Soc., Vol.213, Amer. Math. Soc., Providence, 1979.

17. L Distribution of zeros of orthogonal polynomials, Trans. Amer. Math. Soc. 249 (1979), 341-361.

18. _ Orthogonal polynomials defined by a recurrence relation, Trans. Amer. Math. Soc. 250 (1979), 369-384.

19. F.W.J. Olver, Error bounds for the Liouville-Green (or WKB) approximation, Proc. Cambridge Phylos. Soc. 57 (1961), 790-810.

20. _ Asymptotics and Special Functions, Academic Press, New York, 1974.

21. W. Patula, Growth and oscillations properties of second order linear difference equations, SIAM J. Math. Anal. 10 (1979), 55-61.

22. R. Spigler and M. Vianello, Liouville-Green approximations for a class of linear oscillatory difference equations of the second order, J. Comput. Appl. Math. 41 (1992), 105-116.

23. _ Discrete and continuous Liouville-Green-Olver approximations: A unified treatment via Volterra-Stieltjes integral equations, SIAM J. Math. Anal. 25 (1994), 720-732.

24. _ A survey on the Liouville-Green (WKB) approximation for linear difference equations of the second order, Proc. 2nd Intern. Conf. on Difference Equations and Appl. (Veszprem 1995), Gordon and Breach, 1997, to appear.

25. G. Szegö, Orthogonal Polynomials, Amer. Math. Soc. Coll. Publ., Vol.23, Amer. Math. Soc., New York, $4^{\text {th }}$ ed., 1975.

26. W. Van Assche, Asymptotic properties of orthogonal polynomials from their recurrence formula, I, J. Approx. Theory 44 (1985), 258-276.

27. Asymptotic properties of orthogonal polynomials from their recurrence formula, II, J. Approx. Theory 52 (1988), 322-338.

28. _ Asymptotics for orthogonal polynomials and three-term recurrences. In: Orthogonal Polynomials: Theory and Practice (Ed. P.G. Nevai), NATO ASI Series, Kluwer, Dordrecht, 1990, pp. 435-462.

29. P. Wilmott, $A$ note on the WKB method for difference equations, IMA J. Appl. Math. 34 (1985), 295-302. 
30. S. Wolfram, Mathematica: A System for Doing Mathematics by Computer, $2^{\text {nd }}$ ed., AddisonWesley, Reading, 1993.

31. R. Wong and H. Li, Asymptotic expansions for second-order linear difference equations, J. Comput. Appl. Math. 41 (1992), 65-94.

Dipartimento di Matematica, Università di Lecce, Via Provinciale Lecce-Arnesano, P.O. BoX 193, 73100 LeCCE, ITALY

Current address: Dipartimento di Meccanica e Automatica, Università di "Roma Tre", Via Corrado Segre 60, 00146 ROMA, Italy

E-mail: spigler@caspur.unile.it; spigler@ulam.dmsa.unipd.it

Dipartimento di Matematica Pura e Applicata, Università di Padova, Via Belzoni 7, 35131 PADOVA, ITALY

E-mail: marcov@euler.math.unipd.it 